\title{
"Determinación de cantidad óptima de Fibra de Acero para la elaboración de Hormigón de Cemento Portland para losas de Pavimentos Rígidos
}

\author{
Gino Flor Chávez, MS.c. ${ }^{1}$, A.H.Yánez, ${ }^{2}$, Diana Robalino Cabrera, Ing ${ }^{1}$, Christopher López Chipre, Ing ${ }^{1}$, Fausto \\ Cabrera Montes, Ing ${ }^{1}$ Ing $^{1}$ and Jorge Arroyo Orozco, Ing ${ }^{1}$ \\ ${ }^{1}$ University of Guayaquil, Career of Engineering Civil, Guayaquil, Ecuador, gino.florch@ug.edu.ec; diana.robalinoc@ug.edu.ec; \\ Christopher.lopezc@ug.edu.ec; fausto.cabreram@ug.edu.ec; jorge.arroyoo@ug.edu.ec. \\ ${ }^{2}$ University of Guayaquil, Career of Networking and Telecommunications, Guayaquil, Ecuador, alcibar.yaneze@ug.edu.ec
}

\begin{abstract}
El hormigón aplicado en losas de pavimentos rígidos con módulo de rotura de 4,5 MPa con adición de fibras de acero ha obtenido un importante prestigio por su gran desempeño frente a requisitos cada vez más exigentes del sector de la construcción. El Hormigón Reforzado con Fibra de Acero (HRFA), no es más que hormigón hecho a partir de cementos hidráulicos, áridos finos, áridos gruesos, agua, aditivos y fibras discretas discontinuas (Dramix 80/60), que conlleva a mejoras en el comportamiento físicomecánico. El presente trabajo ha considerado las características tanto del hormigón como de las fibras, y se basará en los lineamientos de las normas nacionales MTOP 2002, NEVI 12, INEN e internacionales ASTM, ACI.

Su caracterización experimental se basa en determinar una resistencia de concreto específica $\left(f^{\prime} c=350 \mathrm{~kg} / \mathrm{cm} 2\right)$ con tamaño máximo nominal de agregado 3/4" y adición de fibra (Dramix 80/60) en probetas cilíndricas y prismáticas (viga), con el objetivo de comprobar el comportamiento físico-mecánico de estos elementos.

Para la ejecución del muestreo se realizó 15 especímenes con diferentes cantidades de adición $(5,10,20,30,40) \mathrm{Kg}$. de fibras Dramix 80/60. Para la propuesta de la dosificación del hormigón, se analiza las diferentes metodologías de diseño como indica la norma ACI, además mediante investigación bibliográfica y experimental, se comprobará la eficiencia en el uso del HRFA.
\end{abstract}

Keywords-Hormigón, Hormigón Reforzado con Fibra de Acero, Ensayos de Hormigón, Pavimentos Rigidos.

\section{INTRODUCCIÓN}

El hormigón convencional resiste potencialmente esfuerzos a compresión, pero tiende a presentar algunos inconvenientes críticamente en presencia de esfuerzos de tracción y flexión; con la adicción de las fibras, hace que las propiedades específicas como resistencia a la tracción del concreto se potencialice [1].

Los hormigones convencionales no se comportan de manera constante todo el tiempo debido a que, al ir deteriorándose, se reduce la serviciabilidad y en algunos casos, este efecto compromete la durabilidad y comportamiento de la estructura. Por dicha razón se plantea adoptar nuevas metodologías para el uso y aplicación de fibras de acero dentro del diseño de hormigón en la construcción de losas, el cual resulta una solución técnica-económica.[18]
La contracción del hormigón se encuentra vinculada directamente con los cambios de temperaturas durante el proceso de fraguado y primera etapa de endurecimiento, sumado a la baja resistencia a la tracción del hormigón convencional, es el principal responsable a la aparición de fisuras, especialmente a edades tempranas debido a una disminución de volumen o deformación del hormigón. En el hormigón endurecido la adición de fibras de acero mejora el comportamiento estructural de las losas de pavimento.

En el Ecuador aún se mantiene el uso de mallas electrosoldadas en la construcción de losas de pavimento rígido, la cual se propone remplazar con el uso de fibras de acero, debido a que en la actualidad no existe parámetros indicados y/o rangos para la dosificación óptima en la cuantía por metro cúbico.

Los gobiernos locales y la población usuaria de las vías generalmente presentan insatisfacción por la corta duración de los pavimentos rígidos, pese a la inversión requerida $\mathrm{y}$ ejecutada no se logra el objetivo deseado de tener una obra dubrable con el tiempo, siendo esta de uso temporal debido a la baja resistencia a flexión del concreto; generando el deterioro prematuro de los pavimentos rígidos.

De esta manera se propone usar fibras de acero comercialmente llamada Dramix 80/60 con diferentes dosificaciones $(5,10,20$, $30,40) \mathrm{kg}$. para obtener una cuantía óptima, que mejore el funcionamiento de las losas de pavimentos, evitando la propagación de fisuras.

La presente investigación se basará en el área de Construcción de pavimentos rígidos de vías urbanas, en la cual según las Normas del Ministerio de Transporte y Obras Públicas (MTOP), se debe usar hormigón con módulo de rotura 4,5 $\mathrm{MPa}$, por lo consiguiente se procederá a comprobar las propiedades físico-mecánica de este elemento en estado fresco como endurecido con las diferentes porcentjaes de fibras de acero, para así obtener la cantidad optima sin perjudicar la trabajabilidad del mismo.

\footnotetext{
Digital Object Identifier (DOI):

http://dx.doi.org/10.18687/LACCEI2019.1.1.363 ISBN:

978-0-9993443-6-1 ISSN: 2414-6390
}

$17^{\text {th }}$ LACCEI International Multi-Conference for Engineering, Education, and Technology: "Industry, Innovation, And Infrastructure for Sustainable Cities and Communities", 24-26 July 2019, Jamaica. 


\section{ANTECEDENTES HISTÓRICOS}

La Humanidad empezó a utilizar diferentes tipos de fibras para la producción de materiales reforzados. Esta implementación inicia en principios del siglo XX, con el desarrollo del asbestocemento, de este modo partió a lo largo del siglo la implementación de más adiciones para diferentes aplicaciones. En inicio del Siglo XX, las fibras de asbesto se utilizaron en el hormigón, en la década de 1950 aparece el concepto de materiales compuestos y el hormigón reforzado con fibras fue el de mayor interés. [2].

Las patentes sobre el uso de pequeñas piezas de acero dentro del hormigón datan de 1913, para 1970 se produce el desarrollo de morteros y los entonces llamados "Hormigones Reforzados con Fibras" (HRF). Desde entonces se ha extendido su campo de aplicación y actualmente existen diversos ejemplos de uso de fibras de acero en hormigón. [3] [4].

En el comienzo se reforzaron hormigones buscando mejorar la resistencia a tracción, la capacidad de control de fisuras y la respuesta frente a acciones dinámicas. Estos tuvieron éxito en campos de aplicación específicos como el revestimiento de túneles y la reparación de pavimentos o tableros de puentes, que cada vez adquieren mayor vigencia. [2] [14].

Durante la última década del siglo $\mathrm{XX}$ aparece un nuevo motivo: el desarrollo de los hormigones de alta resistencia que gracias a su excelente capacidad para sobrellevar condiciones ambientales o de aplicación particulares, atrae la atención de la industria. [2].

\section{El Nacimiento del Hormigón Armado}

La primera patente de hormigón reforzado con fibras de acero se desarrolló en California en 1874 por A. Berard, se basó en una piedra artificial que utilizaba acero granular procedente de desechos para el refuerzo del hormigón. Desde aquel momento han aparecido numerosas patentes.

En 1911 Graham aplicó por primera vez estas fibras para incrementar la resistencia y estabilidad del hormigón armado convencional. Cabe destacar la patente de G. Martin en 1927, que describe la adición de alambres de acero rizados en el hormigón empleado en tuberías. Con el transcurso de los años, la forma de las fibras se ha ido perfeccionando y en las patentes se utilizan parámetros muy similares a los actuales para fibras de acero.

A partir de los años 50 se realizaron múltiples trabajos de investigación sobre hormigones reforzados con fibras de acero son de bajo o medio contenido en carbono, acero inoxidable y de acero galvanizado. Entre los estudios realizados cabe destacar los de Romualdi, Batson y Mandelen 1963.
Las especificaciones estándar, clasificación y definiciones relativas a las fibras de acero, se encuentran normalizados en la Sociedad Americana para Pruebas y Materiales (ASTM) A820/A820M-06 y requisitos básicos en la Norma Ecuatoriana de la Construccion (NEC) -SE-HM 2015

\section{Descripción y funciones de los pavimentos de carreteras}

\section{Pavimentos}

Es un conjunto de capas superpuestas, relativamente horizontales, que se diseñan y cimentan técnicamente con materiales de propiedades pertinentes y compactadas adecuadamente. Estas estructuras se apoyan sobre la subrasante de una vía producida por el movimiento de tierras en el proceso de exploración y que han de soportar los esfuerzos que las cargas repetidas del tránsito le transmiten durante el periodo para el cual es diseñada. [5]

Un pavimento para cumplir sus licitaciones debe reunir los siguientes requisitos:

- Ser resistente a la acción de las cargas impuestas por el tránsito.

- Ser resistente a los agentes de intemperismo.

- Debe ser durable.

- Presentar condiciones adecuadas de drenaje.

- Debe ser económico.

- Debe poseer el color adecuado para evitar reflejos y deslumbramientos.

- Presentar una superficie adaptada a las velocidades previstas de circulación de los vehículos

- Resistente al desgaste producido por el efecto abrasivo de las llantas de los vehículos.

- Debe presentar una regularidad superficial, tanto horizontal como longitudinal. [5]

\section{Pavimentos rígidos}

Son aquellos que esencialmente están constituidos por una losa de concreto hidráulico, apoyada sobre la subrasante o sobre una capa de material seleccionado, llamado subbase del pavimento rígido. [5]

Los pavimentos de concreto trabajan principalmente a flexión por tal motivo se recomienda que su especificación de resistencia sea acorde con ello, por eso el diseño considera resistencia del concreto trabajando a flexión, que se conoce como resistencia a la flexión por tensión $\left(\mathrm{S}^{\prime} \mathrm{c}\right)$ o Módulo de Rotura (MR) normalmente especificada a los 28 días, por lo tanto, el apoyo de las capas subyacentes ejerce poca influencia en el diseño del espesor del pavimento. [5] 


\section{Fisuras}

Se clasifican en fisuras funcionales y estructurales. Las primeras afectan la seguridad y el confort al tránsito, por lo contrario, las segundas afectan la capacidad de carga e implican la vida útil del pavimento.

Las causas que originan la fisuración del hormigón son diversas. Se pueden distinguir aquellas que tienen lugar antes del endurecimiento del hormigón y aquellas que, por el contrario, tienen lugar después. [6] [15].

Antes del endurecimiento del hormigón

En este caso las causas de la fisuración del hormigón son:

- Movimiento durante la ejecución: movimientos del encofrado o de la subbase.

- Plásticas: por retracción plástica o asentamiento plástico.

- Heladas tempranas.

Después del endurecimiento del hormigón

Las causas de la fisuración son las siguientes:

- Físicas: por áridos con retracción, retracción por secado.

- Químicas: por corrosión del acero, reacción áridoálcali o carbonatación.

- Estructurales: por cargas exteriores o deformaciones impuestas.

- Térmicas: por ciclos hielo-deshielo o contracción térmica temprana.

\section{Hormigón reforzado con fibra de acero (HRFA)}

Los hormigones reforzados con fibras de acero se definen como hormigón que incorpora a su composición fibras pequeñas y discretas que se distribuyen aleatoriamente en su masa. [7].

El concepto en que se basa el hormigón reforzado con fibras (HRF) es en cierto modo semejante al del hormigón armado convencional, "cosiendo" las fisuras que pueden producirse y que dejarían a la estructura fuera de servicio. [8].

La diferencia está en que, en lugar de unas pocas barras, de diámetro relativamente grande y orientadas según una dirección determinada, en el HRF el refuerzo está constituido por infinidad de fibras de pequeño diámetro y aleatoriamente orientadas, a las cuales se transfieren los esfuerzos cuando la matriz empieza a fisurarse. [16]

La pérdida de trabajabilidad que la adición de fibras provoca en el hormigón condiciona el contenido máximo de fibras que se puede incorporar en la dosificación y que suele situarse para las fibras de acero, en función de la aplicación de las fibras empleadas.

Por otro lado, suele aplicarse un límite inferior al contenido de fibras, pues las dosificaciones con bajos contenidos de fibras han dejado experiencias negativas en la construcción [9].

\section{Características del hormigón con fibra de acero}

Están constituidos básicamente por los mismos componentes de hormigón tradicional y más la adición de fibras de acero. La inclusión de fibras, así como alterar el comportamiento del hormigón en estado endurecido, también lo hace en estado plástico, por lo que a algunos de los componentes es necesario que tengas condiciones que en los hormigones tradicionales no son necesarias. [8].

En función de la cantidad de fibras que se van a adicionar al hormigón y de la geometría de éstas, el material compuesto tendrá que sufrir ciertas modificaciones respecto de un hormigón tradicional [10]. Estas modificaciones pasan principalmente por una limitación en el tamaño máximo del árido, menores valores de relación grava-arena, mayores cantidades de aditivos reductores de agua, y mayor demanda de finos, entre otros. [8]

\begin{tabular}{|l|r|r|r|}
\hline \multirow{2}{*}{$\begin{array}{c}\text { Componentes de la } \\
\text { mezcla }\end{array}$} & \multicolumn{3}{|l|}{ Tamaño Máximo de árido (mm) } \\
\cline { 2 - 4 } & \multicolumn{1}{l|}{10} & 20 & \multicolumn{1}{l|}{30} \\
\hline Cemento $(\mathrm{Kg} / \mathrm{m} 3)$ & $350-600$ & $300-530$ & $280-415$ \\
\hline Agua/Cemento & $0,35-0,45$ & $0,35-0,50$ & $0,35-0,55$ \\
\hline$\%$ árido fino - grueso & $45-60$ & $45-55$ & $40-50$ \\
\hline$\%$ de aire ocluido & $4-8$ & $4-6$ & $6-5$ \\
\hline
\end{tabular}

Tabla1: Recomendaciones de proporciones para dosificaciones de HRFA. Fuente: [9].

\section{Comportamiento mecánico de hormigón reforzado con fibra de acero}

La incorporación de fibras de acero en el hormigón modifica sus propiedades sea en estado fresco como en estado endurecido [9]. Esto deduce que se modifiquen los criterios de dosificación respecto a los hormigones tradicionales. [17]

El volumen de fibras a incorporar y sus características afectarán notoriamente al tamaño máximo del árido, a la relación gravaarena, a la cantidad de aditivos Superhiperplasitificantes a emplear y a la cantidad de árido fino.

\section{Tipos de Fibras}

Las fibras son empleadas en aplicaciones estructurales en busca de beneficios adicionales en cuanto a reducción de mano de 
obra, incremento de la durabilidad, y reducción o eliminación del refuerzo tradicional. [11].

Son empleadas para el refuerzo de materiales las principales son fibras de acero, vidrio, sintéticas (carbón, nylon, polipropileno, acrílicas, poliéster) y naturales de diverso origen. De todas ellas, son las de acero las de mayor aplicación en el hormigón estructural y sobre las mismas se centrar este trabajo. [10]

Se presentan de secciones variables y onduladas ya sea a lo largo de toda su longitud o sólo en sus extremidades con el fin de aumentar el anclaje fibra-hormigón

\begin{tabular}{|c|c|c|c|c|}
\hline Tipo de fibra & $\begin{array}{c}\text { Resistencia a } \\
\text { tracción } \\
\text { (MPa) }\end{array}$ & $\begin{array}{c}\text { Módulo de } \\
\text { elasticidad } \\
\text { (GPa) }\end{array}$ & $\begin{array}{c}\text { Densidad } \\
\left(\mathrm{kg} / \mathrm{m}^{3}\right)\end{array}$ & $\begin{array}{c}\text { Alargamiento } \\
\text { de rotura (\%) }\end{array}$ \\
\hline Acero & $500-3000$ & 210 & 7800 & 3,5 \\
Acero & 2100 & 160 & 7860 & 3,0 \\
inoxidable & 2000 & 60 & 2700 & 3,6 \\
Vidrio & 3000 & $200-500$ & 1900 & 0,5 \\
Carbono & 900 & 4 & 1100 & $13,0-15,0$ \\
Nilon & $400-800$ & $5-25$ & 900 & $8,0-20,0$ \\
Polipropileno & $700-900$ & 8,2 & 1400 & $11,0-13,0$ \\
Poliester & $5-8$ & 30 & 2300 & - \\
Hormigón & & & & \\
\hline
\end{tabular}

Tabla 2: Tipos de fibra Fuente: [11].

Fibras de acero.

Las fibras de acero son elementos de corta longitud y pequeña sección; que se adicionan al hormigón con el fin de conferirle ciertas propiedades específicas, hechas con alambre de acero estiradas en frio, para rendir una alta Resistencia a la tracción. Con las características necesarias para dispersarse aleatoriamente en una mezcla de hormigón en estado fresco empleando metodologías de mezclado tradicionales. [12].

Según la NEC-SE-HM 2015 los requisitos básicos:

Acabado: Pueden ser de acero negro pulido o galvanizadas.

Resistencia nominal a la tracción: desde 1160 N/mm2 hasta

$1345 \mathrm{~N} / \mathrm{mm} 2$ con tolerancias de $\pm 7,5 \%$ promedio.

Longitudes: Desde $30 \mathrm{~mm}$ hasta $60 \mathrm{~mm}$.

Diámetros: Desde 0,55 $\mathrm{mm}$ hasta $1,05 \mathrm{~mm}$.

\section{METODOLOGÍA}

\section{Generalidades}

Dosificar o diseñar una mezcla de concreto de alta resistencia con adición de fibra metálica es especificar la composición más adecuada y económica de los agregados finos como gruesos, agua, cemento, fibra y aditivos; con el objetivo de alcanzar una mezcla homogénea con una adecuada trabajabilidad, y que al endurecerse cumpla con las características de durabilidad y resistencia necesaria a cumplir para losas de pavimento rígido con $\mathrm{MR}=4,5 \mathrm{MPa}$. [13].
Para determinar las cantidades adecuadas de los componentes de la mezcla hidráulica, se necesitó de varios diseños de prueba, que fueron analizados para cumplir con las propiedades físicomecánico deseada, obteniendo proporciones idóneas para la composición del mismo.

La etapa experimental de la elaboración de especímenes de hormigón de la investigación, se realizó en las instalaciones de la planta del Centro Técnico de Construcciones S.A. (CETECO) y la caracterización de los agregados en el Laboratorio de suelos y materiales Dr. Ing. Arnaldo Ruffilli ubicado en la Universidad de Guayaquil.

Ensayos para caracterización de los componentes del hormigón

Los ensayos que se someten a los agregados que comprenden el hormigón son normalizados para así tener un mejor control en la calidad de los mismos.

\section{Cemento}

En este trabajo se usará cemento portland de Alta resistencia y durabilidad (HE), el cual cumple con las especificaciones según lo indica la norma Instituto Ecuatoriano de Normalizacion INEN 2380 el cual deberá cumplir los requisitos mostrados en la Tabla 3 .

\begin{tabular}{|l|r|r|}
\hline ENSAYO & \multicolumn{1}{|l|}{ UNIDAD } & NORMA \\
\hline Peso Especifico & $(\mathrm{gr} / \mathrm{cm} 3)$ & ASTM C 188-95 \\
\hline Expansión en Autoclave & $(\%)$ & ASTM C 151-00 \\
\hline Fraguado Vicat & $($ minuto $)$ & ASTM C 191-01 \\
\hline Módulo de Finura - Blaine & - & ASTM C 204-84 \\
\hline
\end{tabular}

Tabla 3: Requisitos físicos del cemento $\mathrm{HE}$ Fuente: [3].

Agua

Esta debe cumplir con parámetros que se observan en la Tabla 4 y que son necesarios para su uso en la dosificación de hormigones.

\begin{tabular}{|l|r|r|}
\hline ENSAYO & UNIDAD & \multirow{2}{*}{ NORMA } \\
\hline PH & gr,/ litro \\
\cline { 1 - 2 } Sustancias disueltas & gr,/ litro \\
\cline { 1 - 2 } Sulfatos & gr,/ litro \\
\cline { 1 - 2 } $\begin{array}{l}\text { Sustancias orgánicas solubles en } \\
\text { éter }\end{array}$ & gr,/litro \\
\cline { 1 - 2 } Ion cloro & \multicolumn{1}{|r|}{-} \\
\cline { 1 - 2 } Hidratos de carbono & \\
\hline
\end{tabular}

Tabla 4: Ensayos para caracterización del agua. Fuente: [3].

$17^{\text {th }}$ LACCEI International Multi-Conference for Engineering, Education, and Technology: "Industry, Innovation, And Infrastructure for Sustainable Cities and Communities", 24-26 July 2019, Jamaica. 
Agregados

Los agregados son parte esencial del hormigón conformando aproximadamente el 70\% del mismo, estos áridos deben ser ensayados como lo indica la norma ecuatoriana y estar dentro del rango admisible que estipula el las Especificaciones Generales de camions y Puentes del Ministierio de Transporte y Obras Publicas (M.T.O.P.) capitulo VIII Sección 803) que hace relacion de los ensayos que se deben realizarse a los agregados que se utilizaran en hormigones hidraulicos.

\begin{tabular}{|c|c|c|c|}
\hline \multicolumn{2}{|r|}{ ENSAYOS } & AGREGADO & $\begin{array}{l}\text { AGREGADO } \\
\text { GRUESO }\end{array}$ \\
\hline ASTM C-125 & Módulo de Finura & si & - \\
\hline $\begin{array}{l}\text { ASTM C-128 } \\
\text { ASTMC-127 }\end{array}$ & Absorción & si & si \\
\hline ASTM C-29 & $\begin{array}{l}\text { Peso Volumétrico } \\
\text { Varillado }\end{array}$ & - & si \\
\hline ASTM C-29 & Peso Volumétrico Suelto & si & si \\
\hline ASTM C-29 & $\begin{array}{r}\text { Densidad Saturada } \\
\text { Superficialmente Seca }\end{array}$ & si & si \\
\hline ASTM C-88 & Desgaste a los Sulfatos & - & si \\
\hline ASTM C-131 & $\begin{array}{c}\text { Resistencia a la } \\
\text { degradación e impacto en } \\
\text { la maquina Abrasión de los } \\
\text { Ángeles }\end{array}$ & - & si \\
\hline
\end{tabular}

Fig 1. Ensayos realizado Agregado Fino y Agregado Grueso. Fuente: [3].

\section{Análisis del hormigón \\ En estado fresco}

\section{Medición de temperatura.}

El hormigón en estado fresco debe estar a una temperatura promedio que indica la norma, si este excede de los $32^{\circ} \mathrm{C}$ puede generar futuros problemas como por ejemplo perdida de trabajabilidad y generar fisuras en estado endurecido. (Ver Tabla 6).

\begin{tabular}{|l|l|l|}
\hline ENSAYO & UNIDAD & NORMA \\
\hline Temperatura & ${ }^{\circ} \mathrm{C}$ & ASTM C-1064 \\
\hline
\end{tabular}

Tabla 6: Ensayo para medir temperatura. Fuente: [3].

\section{Medición de contenido de aire - Método de Presión.}

Aplicado en mezclas de hormigón con agregados relativamente densos. Permite determinar la cantidad de aire que pueda tener un hormigón en estado fresco, sin incluir cantidades de aire adicional que puedan contener las partículas de los agregados.

Para la ejecución de este ensayo la muestra, equipo y procedimiento (Ver Tabla 7).

\begin{tabular}{|l|l|l|}
\hline ENSAYO & UNIDAD & NORMA \\
\hline $\begin{array}{l}\text { Contenido de aire - Método de } \\
\text { presión }\end{array}$ & $(\%)$ & ASTM C 231 \\
\hline
\end{tabular}

Tabla 7: Ensayo medición Contenido de aire. Fuente: [3].

Elaboración de especímenes y curado.

Para comprobar la dosificación propuesta con adición de fibra de acero, se procede a realizar el correspondiente muestreo, que conlleva a la elaboración de especímenes tanto para vigas como cilindros de acuerdo a lo especificado en las normas, los cuales por confiabilidad y error que se pueda presentar en la conclusión de resultados, se optó 15 especímenes por cada adición de fibra que se propuso de los cuales 4 cilindros para $\operatorname{los} 7,14$ y 28 dias y los tres restante para asegurar valores por si acaso exista dispersión en los resultados.

Los procedimientos para la elaboración de cada uno conllevan a diferentes pasos a seguir que se concentran en las normas ASTM C-31. Por lo luego de 24 horas aproximadamente se procede a desmoldar y así ser expuesto al curado correspondiente para ser ensayados.

\section{Hormigón en estado endurecido}

\section{Resistencia a compresión de los especímenes elaborados}

Consiste en aplicar una carga axial de compresión a los especímenes a una velocidad constante que indica la norma hasta que se produzca la falla. Los especímenes deben ser extraídos del curado, dejarlo a la intemperie como mínimo una hora antes de ser ensayos.

Para la ejecución de la rotura se observa las caras del cilindro o espécimen, porque si estas varían en más de $0,05 \mathrm{~mm}$. Respecto al plano, deben ser tratadas para obtener su total paralelismo de sus caras y se logra usando mortero de azufre o con almohadillas no adherentes de neopreno. Los especímenes deben tener una relación de altura - diámetro de igual a 2. (Ver Tabla 8)

\begin{tabular}{|l|l|l|}
\hline ENSAYO & UNIDAD & NORMA \\
\hline Resistencia A Compresión & $\mathrm{Kg} / \mathrm{cm}^{2}$ & $\begin{array}{l}\text { ASTM C-39 - INEN } \\
1573\end{array}$ \\
\hline
\end{tabular}

Tabla 8: Norma ensayo de compresión a cilindros. Fuente: [3].

\section{Resistencia a la tracción de los especímenes elaborados}

Este es el ensayo clave en la ejecución de la investigación debido a que hormigón propuesto debe cumplir el Módulo de rotura deseado. La ejecución del este ensayo no conlleva a

$17^{\text {th }}$ LACCEI International Multi-Conference for Engineering, Education, and Technology: "Industry, Innovation, And Infrastructure for Sustainable Cities and Communities", 24-26 July 2019, Jamaica. 
ninguna variación respecto al ensayo de compresión debido a que las probetas de ensayan en estado húmedo.

Pero por lo contrario el equipo para realizar el ensayo cambia por la forma prismática (vigas) y dimensiones de las mismas. Este genera una carga aplicada por la prensa y se distribuye a lo largo de la sección donde tiene contacto la viga y se trasmite como en reacciones iguales a los apoyos de la viga. Al ensayar los especímenes se determina el Módulo de Rotura, así como la deflexión máxima que puede alcanzar el hormigón. El procedimiento de la ejecución de detalla en la norma ASTM C78.

\section{Evaluación de Fisuras}

La ubicación y magnitud de la fisuración, así como el estado general del hormigón de una estructura, se pueden determinar mediante observaciones directas e indirectas, ensayos no destructivos y destructivos, y ensayos de testigos extraídos de la estructura.

En esta Investigación se realizará observación directa e indirecta las cuales se deben registrar las ubicaciones y anchos de las fisuras utilizando un esquema de la estructura. Marcar sobre la superficie de la estructura es útil para ubicar con precisión las fisuras en el esquema.

\section{INTERPRETACION DE RESULTADOS}

\section{Recolección de datos}

Para la elaboración de las muestras, se analizará las características físico-mecánicas de los agregados y de esta forma se especifica la dosificación adecuada para obtener las cantidades óptimas de los materiales.

Análisis de los ensayos para la caracterización de los agregados

\section{Granulometría}

En las gráficas mostradas que el agregado se encuentra dentro del parámetro correspondiente a lo que indica la norma ASTM C-33.

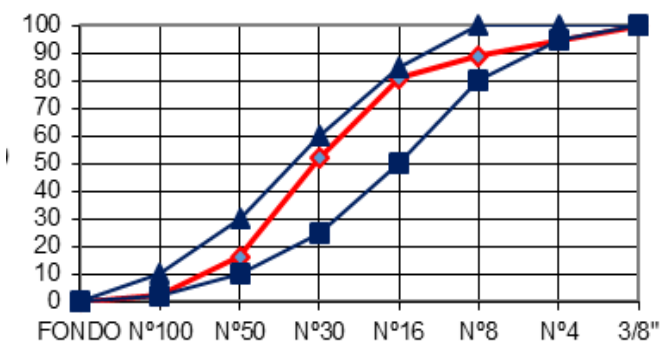

Fig 2: Curva granulométrica del agregado fino Fuente: [3].

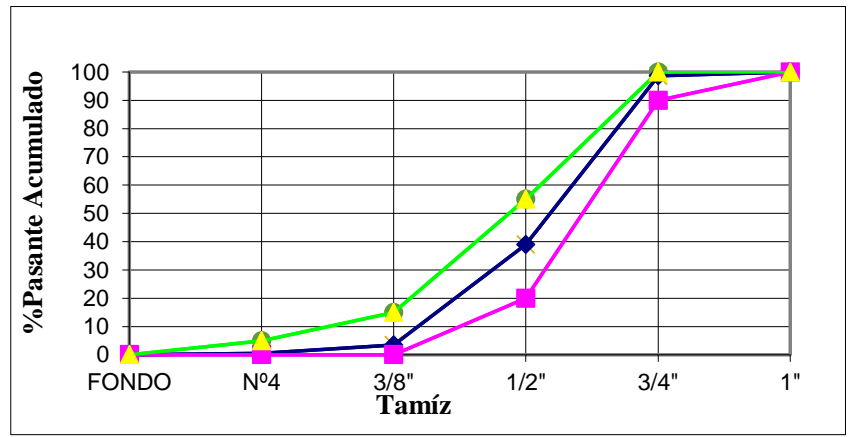

Fig 3: Curva granulométrica del agregado grueso. Fuente: [3].

Siendo de esta manera agregados de óptimas propiedades, además se comprobó el tamaño máximo Nominal del Agregado grueso en la ejecución de este ensayo.

\section{Cuadro de resultados de los ensayos de los agregados}

En la Tabla 9 se presenta los resultados obtenidos de los ensayos realizados a los agregados finos y gruesos, presentando una absorción permitida que no afecta la relación $\mathrm{A} / \mathrm{C}$; el desgaste por abrasión y sulfato de sodio también se encuentra en los rangos permitidos considerando que son de óptima calidad. MTOP Capítulo VIII Sección 801.

\begin{tabular}{|c|c|c|c|}
\hline \multicolumn{2}{|c|}{ ENSAYOS } & $\begin{array}{c}\text { AGREGADO } \\
\text { FINO }\end{array}$ & $\begin{array}{c}\text { AGREGADO } \\
\text { GRUESO }\end{array}$ \\
\hline ASTM C-125 & Módulo de Finura & 2,65 & - \\
\hline $\begin{array}{l}\text { ASTM C-128 } \\
\text { ASTMC-127 }\end{array}$ & Absorción & $1,85 \%$ & $1,42 \%$ \\
\hline ASTM C-29 & $\begin{array}{l}\text { Peso Volumétrico } \\
\text { Varillado }\end{array}$ & - & $1663 \mathrm{~kg} / \mathrm{m}^{3}$ \\
\hline ASTM C-29 & $\begin{array}{ll}\text { Peso } & \text { Volumétrico } \\
\text { Suelto } & \end{array}$ & $1489 \mathrm{~kg} / \mathrm{m}^{3}$ & $1471 \mathrm{~kg} / \mathrm{m}^{3}$ \\
\hline ASTM C-29 & $\begin{array}{l}\text { Densidad Saturada } \\
\text { Superficialmente } \\
\text { Seca }\end{array}$ & $2672 \mathrm{~kg} / \mathrm{m}^{3}$ & $2857 \mathrm{~kg} / \mathrm{m}^{3}$ \\
\hline ASTM C-88 & $\begin{array}{lll}\text { Desgaste } & \text { a } & \text { los } \\
\text { Sulfatos } & & \\
\end{array}$ & - & $0,72 \%$ \\
\hline ASTM C-131 & $\begin{array}{l}\text { Resistencia a la } \\
\text { degradación } \\
\text { impacto en la } \\
\text { maquina Abrasión de } \\
\text { los Ángeles }\end{array}$ & - & $14,80 \%$ \\
\hline
\end{tabular}

Tabla 9: Resultados de los agregados Fuente: [3].

\section{Dosificación propuesta}

Para el diseño del hormigón reforzado con fibras de acero, se tiene la norma del Instituto Americano del Concreto ACI-544 Capitulo 2, "Hormigón Reforzado con fibras acero", en dicha norma se encuentra la Tabla 2.2 "Rango de proporciones para el Hormigón Reforzado con fibras de acero", el inconveniente de la tabla es que solo muestra rangos muy amplios de cantidad de los componentes del concreto y no se especifica la cantidad

$17^{\text {th }}$ LACCEI International Multi-Conference for Engineering, Education, and Technology: "Industry, Innovation, And Infrastructure for Sustainable Cities and Communities", 24-26 July 2019, Jamaica. 
apta para la resistencia deseada de diseño, en este caso losas de pavimentos rígidos.

Cabe indicar que en el ACI-211 se mantiene una secuencia de pasos para el respectivo diseño, por tal motivo, se toma en cuenta el procedimiento de dicha norma para después ajustarlos con respecto los rangos del ACI-544.

\section{Dosificación (ACI-211)}

La dosificación mostrada a continuación cumple con todos los parámetros del diseño de hormigón para losas de pavimentos rígidos, para una resistencia a 28 días, con módulo de Rotura 4,50, MPa y esfuerzo de Compresión f’c $=350 \mathrm{~kg} / \mathrm{cm} 2$.

En la Tabla 10, se observa que la cantidad de cemento varian de acuerdo al diseño que se escoja. Las tres dosifiaciones realizadas están dentro del rango que estipula la ACI- 544 debido que debe de estar entre 300 a $530 \mathrm{~kg} / \mathrm{m} 3$.

Para que la cantidad de cemento cumpla con lo estipulado en la ACI-544, se aplicó los siguientes aditivos:

Superhiperplastificantes (reductor de agua y cemento), obtener mejor trabajabilidad, debido que, según el fabricante de fibras de acero, al añadir fibras al hormigón pierda trabajabilidad.

Retardante, para mantener la trabajabilidad durante 30 minutos como indica la norma según el asentamiento especificado que la tolerancia varíe en menos es de $40 \mathrm{~mm}$.

\begin{tabular}{|l|l|l|l|}
\hline $\begin{array}{l}\text { COMPONENTES } \\
\text { HEL } \\
\text { HORMIGON }\end{array}$ & $\begin{array}{l}\text { Hormigón } \\
\text { Convencional } \\
\text { (ACI 211) (kg) }\end{array}$ & $\begin{array}{l}\text { H. Conv. } \\
\text { (ACI 211) } \\
\text { SHP+R }\end{array}$ & $\begin{array}{l}\text { DISEN +(SHP+R) } \\
\mathbf{5 4 C I}\end{array}$ \\
\hline Cemento & 557,11 & 418,48 & 418,48 \\
\hline Agua & 211,7 & 179,95 & 179,95 \\
\hline Piedra & 989,96 & 1125,78 & 895,29 \\
\hline Arena & 617,24 & 701,92 & 837,32 \\
\hline Retarder & & 0,84 & 0,84 \\
\hline $\begin{array}{l}\text { SuperHiper } \\
\text { Plastificante }\end{array}$ & & 1,26 & 1,26 \\
\hline A/C & 0,38 & 0,43 & 0,43 \\
\hline AF+AG & 1607,19 & 1827,7 & 1732,61 \\
\hline AF/AG (\%) & $\mathbf{3 8 , 4 0 \%}$ & $\mathbf{3 8 , 4 0 \%}$ & $\mathbf{4 8 , 3 3 \%}$ \\
\hline \% Aire & $\mathbf{2 , 0 0 \%}$ & $\mathbf{2 , 0 0 \%}$ & $\mathbf{5 , 0 0 \%}$ \\
\hline & \multicolumn{3}{|l|}{ Fuente: [3]. } \\
\hline
\end{tabular}

Con base a la dosificación de la Tabla 10, diseño ACI 544 $+(\mathrm{SHP}+\mathrm{R})$, el hormigón fue diseñado para un revenimiento de $22 \mathrm{~cm}$ y con un aire ocluido del $5 \%$, el cual se realizarán los ensayos respectivos, sin fibras y con diferentes porcentajes de adición de fibras $(5 \mathrm{~kg} / \mathrm{m} 3,10 \mathrm{~kg} / \mathrm{m} 3,20 \mathrm{~kg} / \mathrm{m} 3,30 \mathrm{~kg} / \mathrm{m} 3$ y 40 $\mathrm{kg} / \mathrm{m} 3$ ), para obtener los resultados requeridos para la determinación de la cuantía óptima. Cabe recalcar que el fabricante indica que la dosificación mínima es $10 \mathrm{~kg} / \mathrm{m} 3$.

\section{Ensayos del hormigon en estado fresco Ensayo de Revenimiento}

Con respecto a los ensayos de hormigón en estado fresco se observa en la Figura 4 que a medida que se incrementa la cantidad de fibras de acero, el hormigón se torna menos trabajable.

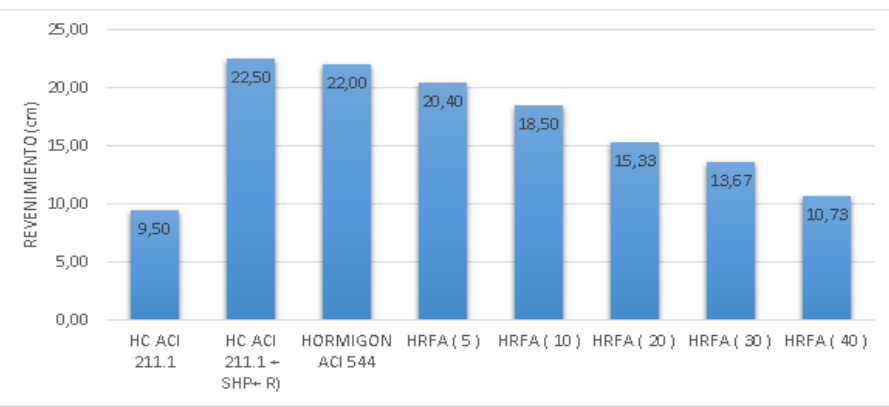

Figura 4: Revenimientos promedio con y sin adicion de fibras Fuente: [3].

\section{Temperatura de la mezcla}

Los resultados de la temperatura del hormigón, están dentro de lo que permite la nomrtiva nacional e internacional que indica que no debe de exceder de $32^{\circ} \mathrm{C}$ tal como se observa en la Tabla 11.

\begin{tabular}{|c|c|c|c|c|}
\hline $\begin{array}{c}\text { TIPO DE } \\
\text { HORMIGON }\end{array}$ & $\begin{array}{c}\text { Toma 1 } \\
{ }^{\circ} \mathrm{C}\end{array}$ & $\begin{array}{c}\text { Toma 2 } \\
{ }^{\circ} \mathbf{C}\end{array}$ & $\begin{array}{c}\text { Toma 3 } \\
{ }^{\circ} \mathbf{C}\end{array}$ & $\begin{array}{c}\text { Temperatura } \\
\text { Promedio } \\
{ }^{\circ} \mathrm{C}\end{array}$ \\
\hline HC ACI 211.1 & 31,50 & 30,10 & 30,17 & $\mathbf{3 0 , 5 9}$ \\
\hline $\begin{array}{c}\text { HC ACI 211.1 } \\
\text { SHP+R) }\end{array}$ & 30,50 & 30,80 & 30,11 & $\mathbf{3 0 , 4 7}$ \\
\hline HORMIGON ACI & 31,18 & 31,80 & 30,11 & $\mathbf{3 1 , 0 3}$ \\
\hline H44 & 31,22 & 31,20 & 30,40 & $\mathbf{3 0 , 9 4}$ \\
\hline HRFA (5) & 30,22 & 30,18 & 30,42 & $\mathbf{3 0 , 2 7}$ \\
\hline HRFA (20) & 30,83 & 30,42 & 30,12 & $\mathbf{3 0 , 4 6}$ \\
\hline HRFA (30) & 30,14 & 31,50 & 30,45 & $\mathbf{3 0 , 7 0}$ \\
\hline
\end{tabular}

Tabla 11: Temperatura del hormigon con y sin adicion de fibras. Fuente: [3].

$17^{\text {th }}$ LACCEI International Multi-Conference for Engineering, Education, and Technology: "Industry, Innovation, And Infrastructure for Sustainable Cities and Communities", 24-26 July 2019, Jamaica. 


\section{Ensayo de Contenido de Aire}

Respecto al contenido de aire en los hormigones elaborados se observa que el hormigón convencional tiene un porcentaje aproximado de aire ocluido del $2 \%$, con respecto al diseño de hormigón reforzado con fibras (HRFA) el aire está en el rango entre 4 y $6 \%$ cumpliendo la norma ACI-544. (Ver Tabla 12)

\begin{tabular}{|c|c|c|c|c|}
\hline $\begin{array}{c}\text { Tipo } \\
\text { De } \\
\text { Hormigón }\end{array}$ & $\begin{array}{c}\% \\
\text { aire }\end{array}$ & $\begin{array}{c}\% \\
\text { aire }\end{array}$ & $\begin{array}{c}\% \\
\text { Aire }\end{array}$ & $\begin{array}{c}\% \text { Aire } \\
\text { Promedio }\end{array}$ \\
\hline HC ACl 211.1 & $1,85 \%$ & $1,80 \%$ & $1,90 \%$ & $1,85 \%$ \\
\hline HC ACl 211.1 + SHP+R & $4,80 \%$ & $4,70 \%$ & $4,60 \%$ & $4,70 \%$ \\
\hline Diseño ACl 544 & $5,10 \%$ & $5,15 \%$ & $5,00 \%$ & $5,08 \%$ \\
\hline HRFA (5) & $4,95 \%$ & $5,00 \%$ & $4,88 \%$ & $4,94 \%$ \\
\hline HRFA (10) & $4,80 \%$ & $4,85 \%$ & $4,80 \%$ & $4,82 \%$ \\
\hline HRFA (20) & $4,55 \%$ & $4,60 \%$ & $4,65 \%$ & $4,60 \%$ \\
\hline HRFA (30) & $4,35 \%$ & $4,30 \%$ & $4,40 \%$ & $4,35 \%$ \\
\hline HRFA (40) & $4,20 \%$ & $4,10 \%$ & $4,00 \%$ & $4,10 \%$ \\
\hline
\end{tabular}

Tabla 12: Contenido de aire promedio con y sin adicion de fibras Fuente: [3].

\section{Ensayos del hormigon en estado endurecido}

\section{Ensayo de Resistencia a flexion}

Una vez obtenida la dosificación y realizado los ensayos al hormigón en estado fresco se elaboraron 15 probetas prismáticas $(15 \times 15 \times 55) \mathrm{cm}$ por cada diseño y adición de fibras, para verificar la resistencia a flexión. En la Tabla 13 se compara la resistencia a flexión del hormigon convencional (HC) y Hormigón con diferentes porcentajes de fibra, observando que a mayor cantidad de fibra aumenta la resistencia. Ademas todos los resultados superan el $100 \%$ de la resistencia requerida a los 28 días.

\begin{tabular}{|c|c|c|c|c|c|c|}
\hline $\begin{array}{l}\text { Edad de la } \\
\text { muestra }\end{array}$ & $\begin{array}{c}\text { HC diseño } \\
\mathrm{ACl}-544+(\mathrm{SHP}+\mathrm{R})\end{array}$ & $\begin{array}{c}+5 \mathrm{~kg} / \mathrm{m3} \\
\text { de fibra }\end{array}$ & $\begin{array}{c}+10 \\
\mathrm{~kg} / \mathrm{m} 3 \mathrm{de} \\
\text { fibra }\end{array}$ & $\begin{array}{c}+20 \\
\mathrm{~kg} / \mathrm{m} 3 \mathrm{de} \\
\text { fibra }\end{array}$ & $\begin{array}{c}+30 \\
\mathrm{~kg} / \mathrm{m} 3 \\
\text { de fibra }\end{array}$ & $\begin{array}{c}+40 \mathrm{~kg} / \mathrm{m} 3 \\
\text { de fibra }\end{array}$ \\
\hline 3 & 2,20 & 2,49 & 2,79 & 2,92 & 3,02 & 3,09 \\
\hline 7 & 3,10 & 3,37 & 3,84 & 3,94 & 3,97 & 4,02 \\
\hline 14 & 4,11 & 4,24 & 4,68 & 4,79 & 4,90 & 5,02 \\
\hline 21 & 4,57 & 4,84 & 5,05 & 5,13 & 5,23 & 5,64 \\
\hline 28 & 5,18 & 5,23 & 5,44 & 6.07 & 6,25 & 6,77 \\
\hline
\end{tabular}

Tabla 13: Ensayo de resistencia a flexion en $H C$ y $H R F A$ Fuente: [3].

\section{Ensayo de deformación}

Las deformaciones máximas en $\mathrm{mm}$ de las vigas sometidas a flexión obteniendo que a mayor contenido de fibra tiende a mayor deformación presentando fisuras sin llegar a la rotura, caso contrario a lo que se denotó en el HC, la cual se fracturó. (Ver Tabla 13)

\begin{tabular}{|c|c|c|}
\hline TIPO DE HORMIGÖN & Carga (MPA) & Deformación (mm) \\
\hline HRFA SIN FIBRA & 5,18 & 1,27 \\
\hline HRFA $(5 \mathrm{Kg})$ & 5,23 & 1,4 \\
\hline HRFA $(10 \mathrm{Kg})$ & 5,44 & 2,41 \\
\hline HRFA $(20 \mathrm{Kg})$ & 6,07 & 3,3 \\
\hline HRFA $(30 \mathrm{Kg})$ & 6,25 & 3,3 \\
\hline HRFA $(40 \mathrm{Kg})$ & 6,77 & 3,94 \\
\hline
\end{tabular}

Tabla 13: Deformaciones máximas admisibles en HC y HRFA. Fuente: [3].

\section{Ensayo de Resistencia a compresión}

En la tabla 14, se compara la resistencia del $\mathrm{HC}$ con los hormigones con diferentes adiciones de fibra observando que existe un incremento de la resistencia al aumentar el porcentaje de fibra.

\begin{tabular}{|c|c|c|c|c|c|c|}
\hline $\begin{array}{c}\text { EDAD DE LA } \\
\text { MUESTRA }\end{array}$ & $\begin{array}{c}\text { Hormigón } \\
\text { diseño ACl } \\
\mathbf{5 4 4}+\mathbf{+ S H P + R )}\end{array}$ & $\mathbf{+ 5} \mathbf{~ k g / m 2}$ & $\mathbf{+ 1 0} \mathbf{~} \mathbf{g} / \mathbf{m 3}$ & $\mathbf{+ 2 0} \mathbf{~ k g} / \mathbf{m 3}$ & $\mathbf{+ 3 0} \mathbf{~ k g} / \mathbf{m 3}$ & $\mathbf{+ 4 0} \mathbf{~ k g} / \mathbf{m 3}$ \\
\hline $\mathbf{3}$ & 215,16 & 217,29 & 223,59 & 231,89 & 239,79 & 240,42 \\
\hline $\mathbf{7}$ & 304,34 & 306,76 & 308,48 & 319,59 & 321,55 & 324,36 \\
\hline $\mathbf{1 4}$ & 371,40 & 373,49 & 388,53 & 394,73 & 400,75 & 405,69 \\
\hline $\mathbf{2 1}$ & 408,12 & 410,42 & 413,74 & 423,72 & 426,67 & 432,44 \\
\hline $\mathbf{2 8}$ & 462,38 & 466,45 & 471,58 & 489,29 & 501,79 & 508,27 \\
\hline
\end{tabular}

Tabla 14: Ensayo de resistencia a compresión en HC y HRFA Fuente: [3].

\section{DISCUSION DE RESULTADOS}

Los agregados gruesos como finos y/o componentes del hormigón cumplen con todos los parámetros que indican las normas.

La adición de fibras al hormigón produce:

\section{Hormigón en Estado Fresco}

La trabajabilidad de la mezcla con adicon de fibra metálica disminuye en comparación del Hormigón Convencional. El HRFA con la cantidad optima disminuye el $30 \%$ de revenimiento.

El porcentaje de aire ocluido disminuye con la adición de fibras aproximadamente $0,5 \%$, lo que se concluye, que, a menor trabajabilidad, menor porcentaje de aire. 
En todos los diseños la temperatura se encontraba debajo del límite permisible por la norma ASTM y/o INEN que es $32^{\circ} \mathrm{C}$.

\section{Hormigón en Estado Endurecido}

La resistencia de diseño a compresión f'c $=350 \mathrm{~kg} / \mathrm{cm} 2$ con fibras presenta un incremento del 5,99\% a los 28 días, de la resistencia especificada, lo que es un incremento despreciable en comparación con el de flexión.

Referente a la resistencia a la flexion, el hormigon reforazado con fibra aumenta más del $35 \%$ de su resistencia de diseño a los 28 días, de igual manera cambia su comportamiento de rotura frágil a una dúctil.

\section{CONCLUSIONES Y RECOMENDACIONES}

La cantidad de adición de fibras de acero optima es de 20 $\mathrm{kg} / \mathrm{m} 3$, para losas de pavimentos con $\mathrm{MR}=4,5 \mathrm{MPa}$.

A mayor cantidad de fibras dismunye la trabajabilidad y aire ocluido.

La resistencia a compression de las mezclas que se adicionaron fibras no crece considerablemente referente con la mezcla convencional, en cambio la rotura a flexion crece considerablemnte respect a la mezclas elaboradas de hormigon convencional.

Las probetas de hormigon reforzado con fibras no permiten la rotura de la viga debido a que provoca un cosido interno de la estructura.

\section{Recomendaciones}

Se recomienda hacer ensayos para obtener una correlación entre la resistencia de flexión y compresión tal como existe en hormigones convencionales que según el ACI-211 es de $10 \%$ al $15 \%$.

De ahí que se propone la realización de una mayor cantidad de probetas con las mismas condiciones que las empleadas para afianzar la veracidad de los presents resultados.

\section{REFERENCIAS}

[1]. FIGUEROA ANDRADE, Manuel. Comparativa de la tenacidad entre hormigón convencional, hormigón reforzado con fibras de acero y hormigón reforzado con fibras de polipropileno. 2013. Tesis Doctoral. Caminos.

[2]. IRIAS PINEDA, Ana Sofia. Refuerzo de elementos estructurales con hormigones con fibras o sólo fibras. 2013. Tesis Doctoral. Caminos.
[3]. ROBALINO CABRERA, Diana Desire; CHIPRE, López; BOLIVAR, Cristopher. Determinación de cantidad óptima de fibra de acero para la elaboración de hormigón de cemento Portland de $\mathrm{MR}=4$, 5 MPA, para losas de pavimentos rígidos. 2018. Tesis de Licenciatura. Universidad de Guayaquil. Facultad de Ciencias Matemáticas y Físicas. Carrera de Ingeniería Civil

[4]. R. K. Greig, R. P. Knowles, K. J. Pearson, J. W. Smith y E. J. Smith, «Un procedimiento para hacer un material de cemento compuesto, reforzado con fibras de vidrio». Gran Bretaña Patente 8417351, 6 Julio 1984.

[5]. MONTEJO FONSECA, Alfonso. Ingeniería de pavimentos para carreteras. 1998.

[6]. VIOLINI, Daniel; PAPPALARDI, Mariano. PATOLOGÍAS EN PAVIMENTOS DE HORMIGÓN A EDAD TEMPRAN A. 2008.

[7]. PINO, Marcelo Cadima; RODRÍGUEZ, Jorge Rosas; TORRICO, Francisco Aguirre. Comportamiento de hormigones reforzados con y sin fibras. Revista Investigación \& Desarrollo, 2006, vol. 1, no 5.

[8]. CÁNOVAS, Manuel Fernández. Hormigones reforzados con fibras de acero. Informes de la Construcción, 1982, vol. 34, no 342, p. 5 17.

[9]. ACI COMMITTEE 544. Report on Fiber Reinforced Concrete.

[10]. JOVELLS, DIEGO; JOSÉ, FERNANDO. Estudio técnico experimental de hormigones con fibras de polipropileno, fibras metálicas y áridos reciclados para su utilización en pavimentos de baja intensidad de tráfico. 2016.

[11]. Anejo 14, EHE, Recomendaciones para la utilizacion de Hormigones con Fibras. 2008

[12]. CABALLERO, Ángel; TURMO CODERQUE, José. Improved construction of medium-span concrete viaducts. En Concrete engineering for excellence and efficiency: proceedings, fib Symposium Prague 2011, Clarion Congress Hotel Prague, Praha, Czech Republic, 8-10 June 2011. Czech Concrete Society, 2011. p. 491-494.

[13]. KOSMATKA, Steven H., et al. Diseño y Control de Mezclas de Concreto, Portland Cement Association, Skokie, Illinois. EE. UU, 2004.

[14]. CARMONA MALATESTA, Sergio; CABRERA CONTRERAS, Manuel. Comportamiento al corte de hormigones reforzado con fibras de acero. Revista ingeniería de construcción, 2009, vol. 24, no 1 , p. 74-94.

[15]. JUÁREZ, C. A., et al. Comportamiento analítico-experimental a cortante en vigas de concreto fibroreforzado. Concreto y cemento. Investigación y desarrollo, 2015, vol. 6, no 2, p. 64-79.

[16]. SÁNCHEZ, J. L., et al. Estudio experimental del comportamiento a compresión de hormigones autocompactantes reforzados con fibras de acero $=$ Experimental study of performance self-compacting concrete reinforced with steel fibers. Anales de Edificación, 2015, vol. 1, no 2, p. 17-26.

[17]. ALVAREZ, Ana Blanco, et al. Comportamiento a flexión de losas de hormigón reforzado con fibras (HRF). En Resúmenes de

$17^{\text {th }}$ LACCEI International Multi-Conference for Engineering, Education, and Technology: "Industry, Innovation, And Infrastructure for Sustainable Cities and Communities", 24-26 July 2019, Jamaica. 
comunicaciones. Asociación Científico-técnica del Hormigón Estructural, 2014. p. 41-42.

[18]. ALBERTI, Marcos, et al. Combinación de fibras de acero de última generación para el refuerzo de hormigón autocompactante. En HAC 2018. V Congreso Iberoamericano de hormigón autocompactable y hormigones especiales. Editorial Universitat Politècnica de València, 2018. p. 97-106.

$17^{\text {th }}$ LACCEI International Multi-Conference for Engineering, Education, and Technology: "Industry, Innovation, And Infrastructure for Sustainable Cities and Communities", 24-26 July 2019, Jamaica. 